\title{
Adaptação à Universidade em Estudantes Universitários: Um Estudo Correlacional
}

\author{
Marco Antônio Pereira Teixeira \\ Universidade Federal do Rio Grande do Sul \\ Graciele Dotto Castro \\ Luciane da Rosa Piccolo \\ Universidade Federal de Santa Maria
}

\begin{abstract}
RESUMO
O objetivo desta pesquisa foi investigar possíveis correlatos da adaptação à universidade em estudantes universitários. Cinco dimensões de adaptação (carreira, pessoal, interpessoal, estudo e institucional) foram correlacionadas com as seguintes variáveis: nível de participação em atividades extracurriculares, apoio familiar percebido em relação à escolha profissional realizada, apoio familiar emocional percebido, nível de interação extra-classe com professores, nível de comportamento exploratório vocacional e ano do curso. Participaram do estudo 342 estudantes de três cursos diferentes, com média de idade de 21,2 anos. A adaptação à universidade foi avaliada através do Questionário de Vivências Acadêmicas - Revisado e as demais variáveis através de instrumentos específicos. Entre os resultados principais, verificou-se que as variáveis exploração de si e nível de interação extra-classe com professores foram as que mais se correlacionaram com todos os cinco indicadores de adaptação, enquanto a exploração do ambiente apresentou correlações mais baixas (e não se correlacionou com a dimensão estudo). $\mathrm{O}$ apoio familiar emocional percebido apresentou uma relação fraca, mas significativa, com a dimensão interpessoal da adaptação. Os resultados são discutidos à luz da literatura sobre o tema.
\end{abstract}

Palavras-chave: adaptação à universidade; vivências acadêmicas; universitários.

\begin{abstract}
Adjustment to University among University Students: A Correlational Study

The purpose of this study was to investigate possible correlates of adjustment to University among University students. Five dimensions of adaptation (career, personal, interpersonal, study, and institutional) were correlated with the following variables: level of participation on extracurricular activities, perceived familial support concerning professional choice, perceived emotional support by parents, level of interaction with faculty members in out-of-classroom contexts, level of vocational exploratory behavior and course level. The participants in the study included 342 students from three different courses, with a mean age of 21,2 years. Adjustment to university was assessed with the Questionário de Vivências Acadêmicas - Revisado (Academic Experiences Questionnaire - Revised), and the other variables were evaluated with specific instruments. Among the main findings, selfexploration and out-of-classroom interaction with faculty members were the variables with the most significant associations with all five dimensions of adaptation to university, while environmental exploration showed weaker correlations (and did not correlate with the study dimension). Perceived emotional support by parents showed a weak, but significant, relationship with the interpersonal dimension of adaptation. Results are discussed considering the literature on the subject.
\end{abstract}

Keywords: adaptation to university; academic experiences; university students.

As experiências vividas por jovens estudantes em virtude do seu ingresso na universidade e o impacto destas experiências sobre a adaptação à universidade e o desenvolvimento psicológico e profissional têm sido foco de atenção recente na pesquisa psicológica bra- sileira (por exemplo, Granado, 2004; Mercuri \& Polydoro, 2003a; Polydoro, Primi, Serpa, Zaroni \& Pombal, 2001; Teixeira \& Gomes, 2004). Contudo, no âmbito internacional, os estudos nessa área já existem há algumas décadas, e têm buscado identificar diver- 
sos fatores que concorrem para a integração dos estudantes à universidade, para o seu desenvolvimento psicológico e, também, para a sua persistência nos cursos (por exemplo, Belfield, Bulloock \& Fielding, 1999; Cabrera, Castañeda, Nora \& Hengstler, 1992; Kuh \& Hu, 2001; Pascarella, 1980; Pascarella \& Terenzini, 2005; Tinto, 1975).

O interesse em estudar a população de estudantes universitários e suas experiências na universidade justifica-se em virtude do crescente número de jovens que ingressa no ensino superior não apenas no Brasil, mas também em diversos outros países. Conhecer melhor a realidade vivida por estes jovens pode auxiliar os gestores em educação a identificar fatores associados não apenas à evasão escolar, mas também a outros aspectos do desenvolvimento psicossocial dos estudantes. Este conhecimento, por sua vez, pode ser usado como referência para a implementação de programas no âmbito universitário que visem à facilitação da integração do estudante à vida acadêmica e à otimização do seu bem-estar psicológico.

Os estudos no campo da adaptação à universidade têm focalizado, em grande parte, aspectos ligados à retenção ou evasão dos cursos superiores (Bardagi, 2007; Cabrera, Castañeda, Nora \& Hengstler, 1992; Gerdes \& Mallinckrodt, 1994; Lehman, 2005; Mercuri \& Polydoro, 2003b; Terenzini, Lorang \& Pascarella, 1981; Tinto, 1975). Em geral, estes trabalhos têm destacado a importância dos aspectos de integração acadêmica e social para se compreender os comportamentos de evasão e permanência de estudantes nas universidades. Tais estudos têm demonstrado que o envolvimento dos alunos com a vida acadêmica, manifestada através de um bom desempenho escolar, de um senso de desenvolvimento intelectual (como a percepção de que a universidade tem ajudado a desenvolver o raciocínio crítico) e de congruência de valores com professores e pares (ou seja, perceber que partilha valores de trabalho com os demais no contexto do curso) contribui para a permanência dos estudantes em seus cursos. Juntamente com estes aspectos mais acadêmicos do desenvolvimento da carreira, a integração social dos alunos durante sua permanência na universidade (relacionada à qualidade e à freqüência de contato com pares e professores e a similaridade de valores em assuntos não acadêmicos) também tem sido identificada como um elemento importante para a persistência na trajetória universitária. Além disso, as experiências extracurriculares (como participação em projetos de extensão e pesquisa), fora de sala de aula, são ainda apontadas como fatores essen- ciais para a formação e a construção da identidade profissional (Capovilla \& Santos, 2001; Fior \& Mercuri, 2003; Kuh, 1995; Kuh \& Hu, 2001; Teixeira \& Gomes, 2004).

No âmbito da integração social, sabe-se que a rede de amizades ou de apoio social que os universitários estabelecem é um aspecto importante para o ajuste ao ensino superior. $\mathrm{O}$ ingresso na vida acadêmica implica muitas vezes mudanças marcantes no cotidiano dos jovens. Para alguns, surge a necessidade de saírem de suas famílias de origem e irem morar longe dos pais, sozinhos ou com outras pessoas. Mesmo aqueles que continuam morando com suas famílias, nas cidades onde estudaram até o ensino médio, também sofrem com essa transição: os amigos de escola deixam de ser vistos todos os dias, e elos afetivos e sociais com os antigos companheiros podem enfraquecer. $\mathrm{O}$ meio acadêmico pode surgir, assim, como um espaço privilegiado para se re-estabelecer uma rede de relações afetivas e de apoio mais próxima e efetiva. Nesse sentido, alguns estudos têm demonstrado que o apoio dos colegas e a capacidade de estabelecer relações de amizade satisfatórias contribuem para o bem-estar psicológico e integração ao ambiente acadêmico (por exemplo, Polydoro \& Primi, 2003; Tao, Dong, Pratt, Hunsberger \& Pancer, 2000).

A literatura indica ainda outros fatores que podem estar associados a um melhor ou pior ajustamento dos jovens à vida universitária. Um deles diz respeito ao apoio emocional da família ou laço afetivo que os estudantes têm com seus pais. Wintre e Yaffe (2000) observaram um papel importante desempenhado pelos pais, ao menos nos casos dos jovens que ainda moravam com suas famílias de origem. A reciprocidade mútua e as freqüentes trocas de idéias com os pais foram fatores que se mostraram associados a menores índices de estresse e de depressão nos estudantes. Em outro trabalho, Beyers e Goossens (2003) investigaram o papel da separação psicológica dos jovens em relação a seus pais e a adaptação à universidade. Os resultados indicaram que a independência em relação aos pais, associada a sentimentos positivos, era um bom preditor do ajustamento. Isso sugere que a autonomia do jovem facilita a sua adaptação, mas apenas quando essa autonomia não está associada a conflitos na relação pais-filho. Dito de outra forma, o sentimento de autonomia só parece ser positivo para o desenvolvimento dos jovens quando associado à percepção de que os pais permanecem como uma espécie de "base segura" com a qual podem contar em caso de 
dificuldade, um resultado já sugerido por outras pesquisas (Dornbusch, 2000).

Além disso, o apoio emocional recebido dos pais pode ter repercussões positivas na adaptação à universidade não apenas por contribuir diretamente para o bem-estar psicológico dos estudantes, mas também por favorecer o envolvimento dos alunos em comportamentos exploratórios vocacionais. De fato, existem evidências de que o sentimento de apoio emocional está associado a índices mais elevados de exploração relacionada às questões vocacionais (Ketterson \& Blustein, 1997; Kracke, 1997). A explicação para este efeito deriva-se da teoria do apego (Ainsworth, 1989), segundo a qual indivíduos mais seguros emocionalmente colocam-se com mais facilidade em situações novas ou desafiadoras, pois respondem com menor ansiedade nestas situações. Assim, explorando mais o ambiente acadêmico e suas oportunidades, os jovens universitários podem aumentar suas chances de adaptação, à medida em que vão conhecendo melhor a si mesmos e o próprio curso, ao mesmo tempo em que desenvolvem suas habilidades e seu senso de auto-eficácia.

No Brasil, ainda é pequeno o número de estudos que enfocam especificamente o tema da adaptação à universidade e, especialmente, a avaliação desse processo. Dois instrumentos nacionais relacionados a esse assunto são a Escala de Integração ao Ensino Superior (Polydoro, Primi, Serpa, Zaroni \& Pombal, 2001) e a Escala de Avaliação da Vida Acadêmica (Vendramini \& cols., 2004). Estas duas escalas, contudo, são menos mencionadas na literatura que o Questionário de Vivências Acadêmicas (Almeida, Soares \& Ferreira, 2002), originalmente desenvolvido em Portugal, e cuja versão reduzida foi adaptada ao contexto brasileiro (Granado, 2004; Granado, Santos, Almeida, Soares \& Guisande, 2005). O Questionário de Vivências Acadêmicas Revisado (QVA-r) avalia cinco grandes áreas de vivências no âmbito universitário: pessoal (ajustamento psicológico e bem-estar geral, com foco na ausência de características associadas a depressão e ansiedade), interpessoal (nível de integração com amigos e percepção de apoio), carreira (decisão e satisfação com a escolha), estudo (nível de organização e compromisso com estudo) e institucional (satisfação e vínculo com a instituição). Estas dimensões emergiram de análises fatoriais do instrumento original, podendo-se considerar que elas constituem um modelo parcimonioso de indicadores do grau de adaptação dos estudantes à vida universitária. Este instrumento e seu modelo vem sendo utilizado em algumas pesquisas nacionais (por exemplo, Bardagi, Paradiso, Lassance \& Souza,
2005; Schleich, 2006), motivo pelo qual foi escolhido para o presente estudo.

Esta pesquisa, portanto, aborda o problema da adaptação à universidade através da avaliação de variáveis que têm sido consideradas relevantes nesse processo. Nesse sentido, privilegia um modelo de adaptação, operacionalizado através do QVA-r, dentro de uma lógica quantitativa, fundamentando-se em evidências de pesquisas empíricas sobre o assunto. Tal recorte buscou estabelecer um enquadre, ainda que limitado, a partir do qual se pudesse estabelecer um diálogo com a literatura a explorar possibilidades. Assim, o objetivo geral deste estudo foi investigar, exploratoriamente, algumas variáveis envolvidas no ajustamento à universidade a partir do modelo proposto pelo Questionário de Vivências Acadêmicas Revisado (QVA-r; Almeida, Soares \& Ferreira, 2002; Granado, 2004; Granado, Santos, Almeida, Soares \& Guisande, 2005). Mais especificamente, buscou-se verificar o padrão de correlações das cinco dimensões do instrumento QVA-r com as seguintes variáveis, sugeridas pela literatura: nível de participação em atividades extracurriculares, apoio familiar percebido em relação à escolha profissional realizada, apoio familiar emocional percebido, nível de interação extra-classe com professores, nível de comportamento exploratório vocacional e ano do curso (anos cursados).

\section{MÉTODO}

\section{Participantes}

Participaram do estudo 342 estudantes universitários de três cursos de graduação da Universidade Federal de Santa Maria (Direito, Psicologia e Veterinária). A amostra foi obtida por conveniência. A média de idade foi de 21,2 anos, com desvio-padrão de 2,30 (55\% da amostra foi composta por mulheres). As idades variaram entre 18 e 29 anos, sendo que $94 \%$ dos participantes tinha até 24 anos. Conforme o ano do curso, os participantes se encontravam assim distribuídos: primeiro ano $(21,6 \%)$, segundo $(19,9 \%)$, terceiro $(22,8 \%)$, quarto $(23,7 \%)$ e quinto $(12 \%)$. Todos os três cursos tinham o mesmo tempo total de duração recomendada (cinco anos), e funcionavam em turno diurno (manhã e/ou tarde).

\section{Instrumento}

Utilizou-se um questionário com informações sobre curso, semestre no curso, sexo e idade, além de 
escalas que mediam as variáveis de interesse da pesquisa, conforme especificado a seguir.

Vivências acadêmicas. Foram avaliadas através da versão brasileira do QVA-r, um instrumento com evidência de validade de construto e bons índices de consistência interna (Granado, 2004). O QVA-r mede cinco dimensões de vivências acadêmicas, já descritas na introdução. Cada item é respondido em uma escala Likert ( 1 - nada a ver comigo, 2 - pouco a ver comigo, 3 - algumas vezes de acordo comigo, 4 - bastante a ver comigo, 5 - tudo a ver comigo).

Nível de participação em atividades extracurriculares. Foi medido através de somatório do número de meses em que os alunos estiveram envolvidos em projetos de pesquisa, de extensão e estágios extracurriculares ao longo do curso.

Apoio familiar percebido em relação à escolha profissional realizada. Foi avaliado através de uma escala de três itens com formato de resposta tipo Likert (1 - discordo totalmente, 2 - discordo, 3 - não concordo nem discordo, 4 - concordo, 5 - concordo totalmente) desenvolvida para este estudo (exemplo: "Minha família apóia a minha escolha profissional"). Escores altos indicam a percepção de uma família que dá suporte à opção do estudante, lhe encoraja a prosseguir nos estudos e demonstra interesse em saber como o estudante está indo no curso.

Apoio familiar emocional percebido. Foi medido com uma escala de quatro itens com formato de resposta tipo Likert (como o anterior) feita para esta pesquisa (exemplo: "Eu percebo que meus pais realmente se importam comigo”). Pontuações elevadas sugerem que o estudante percebe que seus pais gostam de si, além de conversar com os mesmos e poder contar com o apoio parental em caso de alguma dificuldade.

Nível de interação extra-classe com professores. Foi avaliado através de uma escala de três itens com formato de resposta tipo Likert (como o anterior) desenvolvida para este estudo (exemplo: "Tenho tido facilidade para conversar informalmente com os professores do meu curso”). Escores altos indicam que o estudante busca manter conversas com os professores do curso fora da sala de aula e percebe que tais contatos lhe propiciam aprendizados interessantes a respeito da profissão.

Nível de comportamento exploratório vocacional. A avaliação foi feita através das Escalas de Ex- ploração Vocacional para universitários (Teixeira, Bardagi \& Hutz, 2007). As escalas medem duas dimensões do comportamento exploratório vocacional: exploração do ambiente (busca de informações sobre o mundo ocupacional e do trabalho) e exploração de si (reflexão ou autoconhecimento sobre características de personalidade, interesses e outras influências relacionadas à escolha ocupacional). Cada item é respondido de acordo com uma escala Likert (1 - raramente ou nunca, 2 - poucas vezes, 3 - com alguma freqüência, 4 - freqüentemente, 5 - muito freqüentemente ou sempre).

\section{Procedimentos}

A aplicação do questionário foi feita coletivamente em sala de aula, após autorização das coordenações dos cursos e dos professores que se dispuseram a ceder tempo em suas aulas. Os alunos foram informados sobre a natureza da pesquisa e convidados a participar. Antes do preenchimento do questionário foram obtidos termos de consentimento para participação na pesquisa. O projeto de pesquisa foi previamente aprovado por um comitê de ética em pesquisa. O tempo total de aplicação do instrumento foi de cerca de 40 minutos.

\section{Análise dos dados}

As variáveis de interesse da pesquisa foram todas correlacionadas entre si utilizando-se correlações de Pearson.

\section{RESULTADOS}

Foram computados escores para cada uma das escalas utilizadas neste estudo calculando-se a média das respostas dadas aos respectivos itens. Como as respostas em cada item podem variar de 1 até 5 , as médias das escalas também respeitam essa amplitude máxima de variação. A Tabela 1 exibe as médias, desvios-padrão, valores mínimo e máximo obtidos nas escalas usadas na pesquisa, bem como os seus coeficientes de consistência interna (alpha de Cronbach). Por sua vez, a Tabela 2 mostra o padrão de correlações observado entre as variáveis do estudo. Para evitar redundância, estes resultados são comentados na próxima seção do artigo (discussão). 
TABELA 1

Médias, Desvios-Padrão (DP), Mínimo, Máximo e Alpha de Cronbach das Escalas

\begin{tabular}{lccccc}
\hline \multicolumn{1}{c}{ Escalas } & Média & DP & Mín & Máx & Alpha \\
\hline QVA - Carreira & 4,11 & 0,68 & 1,58 & 5,00 & 0,89 \\
QVA - Pessoal & 3,39 & 0,70 & 1,36 & 4,79 & 0,86 \\
QVA - Interpessoal & 3,80 & 0,66 & 1,67 & 5,00 & 0,86 \\
QVA - Estudo & 3,51 & 0,71 & 1,25 & 4,88 & 0,82 \\
QVA - Institucional & 3,72 & 0,63 & 1,25 & 4,88 & 0,72 \\
Apoio familiar à escolha & 4,43 & 0,66 & 1,33 & 5,00 & 0,73 \\
Apoio familiar emocional & 4,42 & 0,64 & 1,50 & 5,00 & 0,81 \\
Exploração do ambiente & 3,80 & 0,65 & 1,90 & 5,00 & 0,87 \\
Exploração de si & 3,56 & 0,66 & 1,36 & 5,00 & 0,85 \\
Interação com professores & 3,25 & 0,94 & 1,00 & 5,00 & 0,80 \\
\hline
\end{tabular}

TABELA 2

Correlações Bivariadas entre as Variáveis Centrais do Estudo

\begin{tabular}{|c|c|c|c|c|c|}
\hline Variáveis & (1) & (2) & (3) & (4) & (5) \\
\hline 1. QVA - Carreira & - & & & & \\
\hline 2. QVA - Pessoal &, $39 * *$ & - & & & \\
\hline 3. QVA - Interpessoal &, $32^{\star \star}$ &, $18^{\star \star}$ & - & & \\
\hline 4. QVA - Estudo &, $34^{\star *}$ &, $43^{\star \star}$ &, $16^{\star \star}$ & - & \\
\hline 5. QVA - Institucional &, $49 \star \star$ &, $20^{\star \star}$ &, $28^{\star \star}$ &, $17^{\star \star}$ & \\
\hline 6. Atividades extracuriculares &, $14^{\star *}$ &, 02 &, 09 &, 09 & $18^{\star \star}$ \\
\hline 7. Apoio familiar à escolha &, $12^{\star}$ &, 06 & ,08 &, 09 & ,05 \\
\hline 8. Apoio familiar emocional &, 06 &, $15^{\star \star}$ &, $19^{\star \star}$ &, 03 &, $13^{*}$ \\
\hline 9. Exploração do ambiente &, $14^{\star \star}$ &,- 02 &, $19^{* *}$ &, $19^{* *}$ &, $13^{*}$ \\
\hline 10. Exploração de si &, $33^{\star \star}$ &, $26^{\star \star}$ &, $30^{* *}$ &, $33^{\star \star}$ &, $21^{\star *}$ \\
\hline 11. Interação com professores &, $37 * *$ &, $25^{\star \star}$ &, $32^{\star \star}$ &, $26^{\star \star}$ &, $36^{\star \star}$ \\
\hline 12. Ano &,$- 13^{\star}$ &,- 05 &,- 10 &,- 01 &, 00 \\
\hline
\end{tabular}

Nota. O zero antes da vírgula foi omitido para facilitar a visualização

${ }^{*} p<0,05 .{ }^{* *} p<0,01$.

\section{DISCUSSÃO}

Este estudo teve por objetivo identificar fatores relacionados à adaptação ao contexto universitário entre estudantes de nível superior. Para isso, variáveis consideradas relevantes para a adaptação, conforme indicação da literatura, foram avaliadas e associadas com as cinco dimensões de vivências acadêmicas propostas no instrumento QVA-r, através de análises correlacionais.

Em primeiro lugar, deve-se notar que as médias observadas em todas as escalas do QVA-r estiveram acima do seu ponto médio (valor 3), o que sugere que os participantes desta pesquisa perceberam-se (em média) pelo menos razoavelmente adaptados ao contexto da universidade. As dimensões carreira e interpessoal foram as que apresentaram médias mais altas, revelando satisfação com o curso e com as relações pessoais estabelecidas no âmbito da universidade. Por outro lado, a dimensão pessoal apresentou a menor média, sugerindo que o ajustamento pessoal e o sentimento de bem-estar dos estudantes não são tão altos quanto a satisfação com o curso e com as relações interpessoais (embora não se possa considerar que os estudantes apresentem dificuldades de ajuste pessoal, pois a média ficou acima do ponto central, o que sugere ajuste, e não desajuste psicológico).

Em relação às outras variáveis consideradas, verificou-se que os apoios familiares (tanto à escolha quanto emocional) foram as variáveis com médias mais elevadas, o que mostra que os participantes deste estudo perceberam-se fortemente apoiados por seus familiares. Ainda, os estudantes indicaram exibir comportamentos exploratórios vocacionais (do ambiente e de si) com alguma freqüência (médias entre 3 e 
4 na escala de respostas). A variável com menor média foi o nível de interação com professores, mas ainda assim acima do ponto central, sugerindo que os participantes tinham um nível moderado de conversas e contatos extra-classe com os professores.

$\mathrm{Na}$ análise correlacional, observaram-se diversas correlações estatisticamente significativas, embora a magnitude dos valores tenha sido fraca $($ até 0,30$)$ ou tendendo a moderada (entre 0,30 e 0,49). Ainda que, em termos estatísticos, isso signifique que a capacidade de uma variável "explicar" a outra tenha sido modesta, variando entre aproximadamente $1 \%$ e $25 \%$ (variância compartilhada em cada par de variáveis), tais resultados sugerem relações de interesse teórico e com potencial para instigar novas pesquisas, motivo pelo qual são discutidas aqui.

As correlações observadas entre as dimensões do QVA-r foram todas positivas e estatisticamente significativas. Tal resultado é coerente com o que se poderia esperar, e indica que a adaptação ao contexto universitário depende de múltiplos fatores que estão mutuamente relacionados. Observe-se que a dimensão carreira obteve uma correlação mais forte com a dimensão institucional $(0,49)$, sugerindo que a avaliação que o sujeito faz da satisfação com sua escolha e curso, através do QVA-r, relaciona-se mais diretamente com a avaliação global que o indivíduo faz da instituição na qual estuda (pelo menos quando comparado às outras variáveis). Porém, deve-se considerar que as outras dimensões também apresentaram uma correlação positiva com a dimensão carreira, mostrando a importância dos aspectos de ajuste pessoal $(0,39)$, estudo $(0,34)$ e interpessoal $(0,32)$ para o senso de satisfação com a carreira escolhida. De especial interesse é a correlação da dimensão interpessoal com a dimensão carreira, indicando a importância das amizades estabelecidas no meio universitário para o sentimento de satisfação com o curso e a carreira. Provavelmente, os elos afetivos estabelecidos com os pares no âmbito do curso possibilitam uma maior identificação mútua entre os estudantes, favorecendo a formação ou consolidação da identidade profissional e a persistência no curso (Pascarella \& Terenzini, 2005).

As dimensões pessoal e estudo, por sua vez, correlacionaram-se mais fortemente uma com a outra $(0,43)$. Embora a direcionalidade dos efeitos não tenha sido avaliada, é plausível supor que é o ajuste pessoal a variável que afeta a capacidade do estudante se organizar nos estudos (mais do que o contrário). Além disso, ambas as variáveis mostraram-se associadas com a dimensão carreira, sugerindo uma influência recíproca entre estas variáveis.

As variáveis atividades extracurriculares, apoio familiar à escolha, exploração do ambiente, exploração de si, interação com professores e ano de curso também mostraram-se associadas à dimensão carreira. A correlação com atividades exracurriculares $(0,14)$, embora fraca, confirma os achados de outros estudos que mostram a importância de atividades não curriculares de formação para o desenvolvimento do senso de identidade profissional. Tais atividades possivelmente propiciam o desenvolvimento da auto-eficácia profissional e auxiliam, dessa forma, a consolidar um sentimento de satisfação com a profissão e o curso escolhidos (Capovilla \& Santos, 2001; Fior \& Mercuri, 2003; Teixeira \& Gomes, 2004).

A correlação do apoio parental percebido à escolha profissional com a dimensão carreira, ainda que de pequena magnitude $(0,12)$, indica que tal apoio é um fator que apresenta alguma relevância no processo de adaptação à universidade, pois possivelmente facilita o processo de identificação do estudante com a profissão (provavelmente estimulando-o a envolver-se com o curso, suas atividades e professores), o que pode não ocorrer com jovens que percebem que sua escolha não corresponde às expectativas parentais. Por sua vez, a exploração vocacional do ambiente também se mostrou associada à dimensão carreira, não obstante a força da correlação também tenha sido modesta $(0,14)$. De fato, a exploração do ambiente permite ao estudante ampliar a sua visão da profissão, aumentando as suas possibilidades de construir um projeto profissional mais adequado às suas aspirações. Contudo, as informações obtidas sobre o mundo profissional resultantes da exploração do ambiente parecem não ser suficientes para o desenvolvimento de um senso de identidade de carreira. É necessária uma auto-reflexão que integre tais informações com o autoconceito, valores e a história de vida pessoal. Essa reflexão caracteriza a exploração de si - a outra faceta da exploração vocacional que se mostrou correlacionada com a dimensão carreira (inclusive com maior força - 0,33). Isso sugere que os esforços cognitivos empreendidos pelo próprio sujeito na tentativa de construir um sentido para as suas experiências no contexto universitário são um elemento importante no processo de desenvolvimento da identidade profissional.

Outra variável que se mostrou correlacionada com a dimensão carreira foi o nível de interação extraclasse com professores $(0,37)$, indicando que o contato informal dos alunos com os professores parece ser 
um aspecto importante para o desenvolvimento da identidade profissional (ou pelo menos "de curso") do estudante e de uma conseqüente satisfação com a escolha. Conforme Pascarella e Terenzini (2005), tal tipo de contato tem efeitos positivos sobre o desenvolvimento dos estudantes, tanto em termos cognitivos como também em atitudes e valores, contribuindo para a persistência nos cursos e influenciando o nível de aspiração dos alunos. Os autores apontam ainda que vários estudos mostram que a simples percepção dos estudantes acerca do interesse e da disponibilidade dos professores já é suficiente para aumentar a adesão ao curso. Assim, da mesma forma como ocorre com a interação com os pares, a interação com professores parece promover uma maior aderência aos valores associados ao curso e à profissão, contribuindo para a satisfação com a escolha.

De modo surpreendente, o ano de curso apresentou uma correlação negativa com a dimensão carreira (ainda que pequena: -0,13). Seria mais coerente esperar-se que, com o passar do tempo, o envolvimento e a identificação do aluno com o curso e a profissão fossem de fato aumentando, o que implicaria uma relação positiva entre essas variáveis. É possível que esse efeito inesperado deva-se ao fato de que a avaliação da identificação com o curso e a profissão pode envolver um forte aspecto de idealização por parte dos alunos. Assim, no início do curso escores elevados na dimensão carreira podem significar não uma satisfação com a escolha baseada em critérios realistas, e sim uma expectativa positiva frente ao que está por vir, mas que não foi ainda experimentado. Se assim for, é compreensível que a satisfação com a escolha decline com o tempo, na medida em que as limitações do curso e da profissão vão se tornando aparentes e frustrações vão surgindo (Lassance \& Gocks, 1995; Lassance, Gocks \& Francisco, 1993). Tal decréscimo, contudo, refletiria um ajuste das expectativas à realidade, e não necessariamente um desajustamento com o curso ou com a escolha profissional. Esse é um aspecto metodológico que precisa ser considerado em futuras pesquisas, especialmente quando se utilizar o QVA-r para avaliar a adaptação à universidade.

Em relação à dimensão pessoal, as variáveis que se mostraram correlacionadas foram: apoio emocional dos pais, interação com professores e exploração de si (todas positivamente). A correlação com o apoio emocional familiar, apesar de ser baixa $(0,15)$, correspondeu às expectativas, estando de acordo com evidências que mostram que um elo emocional positivo com a família é importante para o desenvolvimento e ajusta- mento psicológicos de jovens universitários (Lapsley, Rice \& Fitzgerald, 1990; Mounts, 2004). Já a correlação com interação com professores $(0,25)$ sugere um sentido causal inverso (embora deva-se salientar que o delineamento do estudo não permite inferir causalidade): possivelmente, os estudantes que gozam de um melhor ajustamento psicológico sentem-se mais dispostos a interagir com os outros, inclusive professores. Por outro lado, alunos que estejam enfrentando alterações de humor, dificuldades de concentração e ansiedade podem evitar os contatos sociais e assim não estabelecer trocas informais com os professores. É possível, ainda, que os próprios professores mostremse menos disponíveis para atender estudantes que apresentem algum tipo de desajustamento pessoal, especialmente se este desajustamento se refletir em comportamentos que provoquem conflitos de relacionamento ou demonstrem desinteresse pelo aprendizado (o que retro-alimentaria o ciclo de desadaptação).

No âmbito interpessoal, os resultados confirmaram a idéia de que o apoio emocional da família é um elemento associado ao desenvolvimento de boas relações interpessoais (Mounts, Valentiner, Anderson \& Boswell, 2006; Wiseman, Mayseless \& Sharabany, 2006). Embora a correlação tenha sido modesta $(0,15)$, a relação entre estas variáveis é coerente com a perspectiva da teoria de apego (Ainsworth, 1989). Possivelmente, jovens que se percebem apoiados emocionalmente por suas famílias experimentaram este apoio durante toda a vida, o que deve ter contribuído para o desenvolvimento da capacidade de enfrentar a ansiedade envolvida na separação do ambiente familiar e no estabelecimento de novas relações que caracteriza o meio universitário. Dessa forma, os estudantes que percebem uma base emocional segura na família provavelmente são mais seguros de si e mais competentes socialmente, o que se reflete numa maior integração social no contexto universitário.

Note-se que o apoio emocional percebido da família também apresentou uma correlação fraca com a dimensão pessoal, como já comentado anteriormente. Tomados em conjunto, estes resultados sugerem uma relação indireta do apoio emocional familiar sobre a adaptação à universidade. A família pode contribuir para a adaptação não apenas através de um apoio explícito à escolha (o que se verificou na correlação da percepção de apoio à escolha com a dimensão carreira), mas também oferecendo um ambiente acolhedor e emocionalmente continente que promova o desenvolvimento da auto-confiança e da auto-estima durante a 
infância e adolescência, o que repercute positivamente na capacidade adaptativa do sujeito na vida adulta.

Também apareceram como fatores associados à dimensão interpessoal a exploração de si $(0,30)$, a exploração do ambiente $(0,19)$ e a interação com professores $(0,32)$. Este resultado indica que os estudantes que apresentam mais comportamentos exploratórios e que se relacionam mais com os professores fora da sala de aula sentem-se mais satisfeitos com suas interações sociais no âmbito da universidade e percebemse mais competentes nas mesmas. Embora seja difícil estabelecer relações de causalidade entre estas variáveis, pode-se pensar que os comportamentos exploratórios (e a interação com professores é, de alguma forma, também um comportamento exploratório) são um precursor da adaptação interpessoal, na medida em que proporcionam experiências de auto-reflexão e de contato com outras pessoas, oportunizando a ampliação da rede social e o aprendizado de habilidades interpessoais (Bohnert, Aikins \& Edidin, 2007). Essa interpretação é coerente com a teoria da auto-eficácia de Bandura (1997), a qual postula que as próprias experiências pessoais são uma das fontes da auto-eficácia. Assim, pode-se supor que sujeitos mais exploradores têm mais oportunidades de desenvolver suas habilidades sociais e, por conseqüência, desenvolvem uma maior confiança em si mesmos no que diz respeito às relações interpessoais e percebem-se mais satisfeitos nesse aspecto. Outra possibilidade é que as variáveis envolvidas sejam ainda influenciadas por um outro fator comum. Por exemplo, uma atitude pró-ativa e otimista dos sujeitos frente à vida de um modo geral poderia explicar tanto a exploração quanto a interação com professores e a satisfação com as relações interpessoais no contexto da adaptação à universidade.

A dimensão estudo também se mostrou correlacionada com as variáveis exploração do ambiente $(0,19)$, exploração de si $(0,33)$ e interação com professores $(0,26)$. Esta dimensão trata do envolvimento do estudante com as exigências acadêmicas e da sua capacidade de gerir essas responsabilidades. É possível que os estudantes mais exploradores e que interagem informalmente com os professores sejam também mais motivados para o cumprimento das atividades escolares. Assim, a motivação para envolver-se com as questões acadêmicas e vocacionais poderia ser um fator que explicaria a correlação entre estas variáveis.

De modo similar, a dimensão institucional exibiu correlações significativas com exploração do ambiente $(0,13)$, exploração de si $(0,21)$ e interação com professores $(0,36)$. Neste caso, pode-se supor que os comportamentos exploratórios e a interação com professores sejam precursores do sentimento de satisfação com a instituição, pois possibilitam ao aluno um maior envolvimento com a mesma. Na medida em que este envolvimento possa resultar em conseqüências percebidas como positivas pelo indivíduo, seu nível global de satisfação com a instituição tenderia, então, a aumentar. Vale ressaltar que a correlação mais alta observada foi com a variável interação com professores, sugerindo a importância deste aspecto da vida acadêmica para o senso de vinculação do aluno à instituição, o que já foi referido anteriormente e é sugerido pela literatura (Pascarella \& Terenzini, 2005). Ainda, a dimensão institucional apresentou uma correlação pequena $(0,13)$, mas significativa, com o apoio emocional familiar. Este é um resultado difícil de interpretar, mas que é coerente com os demais resultados observados: se o apoio emocional favorece os comportamentos exploratórios e estes o envolvimento com a vida acadêmica, então alguma correlação entre o apoio emocional e a satisfação com a instituição poderia ser esperada.

De um modo geral, portanto, os resultados deste estudo confirmam alguns achados da literatura e apontam para algumas variáveis que merecem uma maior atenção em futuros estudos sobre a adaptação à vida universitária. A associação entre o apoio emocional da família e o desenvolvimento de competências interpessoais, por exemplo, é documentada na literatura internacional conforme citado anteriormente, mas não havíamos encontrado estudos brasileiros que tivessem verificado essa relação em amostras de universitários. Da mesma forma, as consistentes correlações observadas entre as diversas dimensões do QVA$r$ e as variáveis exploração de si e interação extraclasse com professores instigam novas pesquisas. Como se dá o processo de auto-reflexão que constitui a exploração de si ao longo dos anos universitários? De que forma e em que medida esta reflexão leva a uma consolidação da identidade profissional (associada ao curso) ou então à busca por novas possibilidades? Quais fatores, individuais e contextuais, favorecem este tipo de comportamento? De modo similar, a importância da interação com professores para a adaptação à universidade encontrada neste estudo (e em estudo prévios também) aponta para a necessidade de uma investigação mais detalhada desta relação. Seria importante especificar que aspectos dessas interações são mais relevantes para o sentimento de adaptação. Por exemplo, até que ponto a proximidade afetiva percebida pelos alunos em relação aos professores 
contribui para a adaptação? Conversas sobre assuntos não relacionados ao curso ou profissão são também importantes nesse sentido? Em que contextos específicos se dão essas interações extra-classe? Estas são algumas questões que podem ser exploradas no futuro.

Por fim, algumas limitações desta pesquisa precisam ser consideradas. A primeira delas diz respeito à natureza correlacional do estudo. Assim sendo, embora muitas vezes as interpretações oferecidas sugiram certo sentido causal nas relações entre as variáveis, é importante lembrar que o delineamento utilizado não permite testar relações de causalidade, o que faz com que as considerações aqui tecidas precisem ser tomadas apenas como possibilidades. Contudo, deve-se levar em conta que, em virtude dos múltiplos fatores associados à adaptação à universidade, é de se esperar que as variáveis envolvidas encontrem-se, em maior ou menor grau, relacionadas entre si, possivelmente influenciando-se reciprocamente. Isso torna a busca por relações causais simples ou lineares um esforço que provavelmente não refletiria a complexidade do fenômeno. Nesse sentido, cabe também fazer uma ressalva em relação ao modelo de adaptação à universidade usado neste estudo e as variáveis correlatas investigadas. As cinco dimensões do QVA-r, embora abrangentes, com certeza não cobrem todo o espectro de experiências relacionadas à vida universitária. Outro ponto que deve ser levado em consideração na interpretação dos resultados são as magnitudes das correlações obervadas nesta pesquisa, que em geral foram baixas ou moderadas. Se, por um lado, tais correlações indicam variáveis que de algum modo estão associadas à adaptação, a baixa proporção de varição partilhada entre elas sugere que outros fatores, não investigados neste estudo, podem explicar melhor 0 processo de ajustamento à universidade. Enfim, mais pesquisas sobre o assunto são necessárias, incluindo novas variáveis ou mesmo abordando o tema a partir de outras perspectivas, de modo que se possa ampliar a compreensão do fenômeno da adaptação à universidade no contexto brasileiro.

\section{REFERÊNCIAS}

Ainsworth, M. D. (1989). Attachments beyond infancy. American Psychologist, 44, 709-716.

Almeida, L. S., Soares, A. P. C., \& Ferreira, J. C. (2002). Questionário de Vivências Acadêmicas (QVA-r): Avaliação do ajustamento dos estudantes universitários. Avaliação Psicológica, 2, 81-93.

Bandura, A. (1997). Self-efficacy: The exercise of control. New York: W. H. Freeman.
Bardagi, M. P. (2007). Evasão e comportamento vocacional de universitários: Estudos sobre o desenvolvimento de carreira na graduação. Tese de doutorado não-publicada, Universidade Federal do Rio Grande do Sul, Porto Alegre.

Bardagi, M. P., Paradiso, A. C., Lassance, M. C. P., \& Souza, M. S. (2005). Integração acadêmica e neuroticismo em estudantes moradores de residência universitária. Em Association Internationale d'Órientation Scolaire et Professionelle (AIOSP) (Org.), CD AIOSP International Conference (full works): $\mathrm{Ca}$ reers in context - New challenges and tasks for guidance and counseling. Lisboa: AIOSP.

Belfield, C. R., Bullock, A. D., \& Fielding, A. (1999). Graduates’ views on the contribution of their higher education to their general development: A retrospective evaluation for the United Kingdom. Research in Higher Education, 40, 409-438.

Beyers, W., \& Goossens, L. (2003). Psychological separation and adjustment to university: Moderating effects of gender, age, and perceived parenting style. Journal of Adolescent Research, 18, 363-382.

Bohnert, A. M., Aikins, J. W., \& Edidin, J. (2007). The role of organized activities in facilitating social adaptation across the transition to college. Journal of Research on Adolescence, 22, 189-208.

Cabrera, A. F., Castañeda, M. B., Nora, A., \& Hengstler, R. (1992). The convergence between two theories of college persistence. Journal of Higher Education, 63, 143-164.

Capovilla, S. L., \& Santos, A. A. A. (2001). Avaliação da influência de atividades extramuros no desenvolvimento pessoal de universitários. Psico-USF, 6, 49-58.

Dornbusch, S. (2000). Transitions from adolescence: A discussion of seven articles. Journal of Adolescent Research, 15, 173-177.

Fior, C. A., \& Mercuri, E. (2003). Formação universitária: O impacto das atividades não obrigatórias. Em E. Mercuri \& S. A. J. Polydoro (Orgs.), Estudante universitário: Características e experiências de formação (pp. 129-154). Taubaté: Cabral.

Gerdes, H., \& Mallinckrodt, B. (1994). Emotional, social, and academic adjustment of college students: A longitudinal study of retention. Journal of Counseling and Development, 72, 281288.

Granado, J. I. F. (2004). Vivência acadêmica de universitários brasileiros: Um estudo de validade e precisão do QVA-r. Dissertação de mestrado não-publicada, Universidade São Francisco, Itatiba.

Granado, J. I. F., Santos, A. A. A., Almeida, L. S, Soares, A. P., \& Guisande, M. A. (2005). Integração acadêmica de estudantes universitários: Contributos para a adaptação e validação do QVA-r no Brasil. Psicologia e Educação, 1, 33-43.

Ketterson, T. U., \& Blustein, D. L. (1997). Attachment relationships and the career exploration process. Career Development Quarterly, 46, 167-178.

Kracke, B. (1997). Parental behaviors and adolescents' career exploration. Career Development Quarterly, 45, 341-350.

Kuh, G. D. (1995). The other curriculum: Out-of-class experiences associated with student learning and personal development. The Journal of Higher Education, 66(2), 123-155. 
Kuh, G. D. \& Hu, S. (2001). The effects of student-faculty interaction in the 1990s. The Review of Higher Education, 24(3), 309-332.

Lapsley, D. K., Rice, K. G., \& Fitzgerald, D. P. (1990). Adolescent attachment, identity, and adjustment to college: Implications for the continuity of adaptation hypothesis. Journal of Counseling and Development, 68, 561-565.

Lassance, M. C. P., \& Gocks, A. (1995). A formação da identidade profissional em universitários: A questão da prática. Em Associação Brasileira de Orientação Profissional (Org.), Anais do II Simpósio Brasileiro de Orientação Vocacional e Oupacional (pp. 65-70). São Paulo: ABOP.

Lassance, M. C. P., Gocks, A., \& Francisco, D. J. (1993). Escolha profissional em estudantes universitários: Estilos de escolha. Em Associação Brasileira de Orientação Profissional (Org.), Anais do I Simpósio Brasileiro de Orientação Vocacional e Oupacional (s/p). São Paulo: ABOP.

Lehman, Y. P. (2005). Estudo sobre a evasão universitária: As mudanças de paradigma na Educação e suas conseqüências. Tese de Livre-Docência não-publicada, Universidade de São Paulo, São Paulo.

Mercuri, E., \& Polydoro, S. A. J. (Orgs.) (2003a). Estudante universitário: Características e experiências de formação. Taubaté: Cabral.

Mercuri, E., \& Polydoro, S. A. J. (2003b). O compromisso com o curso no processo de permanência/evasão no ensino superior: Algumas considerações. Em E. Mercuri \& S. A. J. Polydoro (Orgs.), Estudante universitário: Características e experiências de formação (pp. 219-236). Taubaté: Cabral.

Mounts, N. (2004). Contributions of parenting and campus climate to freshmen adjustment in a multiethnic sample. Journal of Adolescent Research, 19, 468-491.

Mounts, N. S., Valentiner, D. P., Anderson, K. L., \& Boswell, M. K. (2006). Shyness, sociability, and parental support for the college transition: Relation to adolescents' adjustment. Journal of Youth and Adolescence, 35(1), 71-80.

Pascarella, E. T. (1980). Student-faculty informal contact and college outcomes. Review of Educational Research, 50, 545595.

Pascarella, E. T., \& Terenzini, E. T. (2005). How college affects students: A third decade of research - Vol. 2. San Francisco: Jossey-Bass.

Polydoro, S. A. J., \& Primi, R. (2003). Integração ao ensino superior: Explorando sua relação com características de personali- dade e envolvimento acadêmico. Em E. Mercuri \& S. A. J. Polydoro (Orgs.), Estudante universitário: Características e experiências de formação (pp. 41-66). Taubaté: Cabral.

Polydoro, S. A. J., Primi, R., Serpa, M. de N. da F., Zaroni, M. M. H., \& Pombal, K. C. P. (2001). Desenvolvimento de uma Escala de Integração ao Ensino Superior. Psico-USF, 6, 11-17.

Schleich, A. L. R. (2006). Integração na educação superior e satisfação acadêmica de estudantes ingressantes e concluintes. Dissertação de mestrado não-publicada, Universidade Estadual de Campinas.

Tao, S., Dong, Q., Pratt, M. W., Hunsberger, B., \& Pancer, S. M. (2000). Social support: Relations to coping and adjustment during the transition to university in the People's Republic of China. Journal of Adolescent Research, 15, 123-144.

Teixeira, M. A. P., \& Gomes, W. B. (2004). Estou me formando... E agora? Reflexões e perspectivas de jovens formandos universitários. Revista Brasileira de Orientação Profissional, 5(1), 47-62.

Teixeira, M. A. P., Bardagi, M. P., \& Hutz, C. S. (2007). Escalas de Exploração Vocacional (EEV) para universitários. Psicologia em Estudo, 12, 195-202.

Terenzini, P. T., Lorang, W. G., \& Pascarella, E. T. (1981). Predicting freshman persistence and voluntary dropout decisions: A replication. Research in Higher Education, 15, 109-127.

Tinto, V. (1975). Dropout form higher education - Theoretical synthesis of recent research. Review of Educational Research, 45, 89-125.

Vendramini, C. M. M., Santos, A. A. A., Polydoro, S. A. J., Sbardelini, E. T. B., Serpa, M. N. F., \& Natário, E. G. (2004). Construção e validação de uma escala sobre avaliação da vida acadêmica (EAVA). Estudos de Psicologia, 9, 259-268.

Wintre, M. G., \& Yaffe, M. (2000). First-year students' adjustment to university life as a function of relationships with parents. Journal of Adolescent Research, 15, 9-37.

Wiseman, H., Mayseless, O., \& Sharabany, R. (2006). Why they are lonely? Perceived quality of early relationships with parents, attachment, personality predispositions and loneliness in first-year university students. Personality and Individual Differences, 40, 237-248.

Recebido: 05/03/2007 Última revisão: 20/11/2007 Aceite final: 27/11/2007

\section{Sobre os autores:}

Marco Antônio Pereira Teixeira: Professor no Instituto de Psicologia da Universidade Federal do Rio Grande do Sul.

Graciele Dotto Castro e Luciane da Rosa Piccolo: Alunas do curso de graduação em Psicologia da Universidade Federal de Santa Maria.

Endereço para correspondência: Marco Antônio Pereira Teixeira - Rua Álvares Cabral, 158 - Porto Alegre/RS - CEP 91350-250. Endereço eletrônico: mapteixeira@yahoo.com.br. 